\title{
Usando sensores magnéticos em um trilho de ar
}

(Using magnetic sensor in air's trail)

\author{
Francisco Laudares, Marcia C.S.M. Lopes ${ }^{2}$ e Frederico A.O. Cruz ${ }^{3}$ \\ ${ }^{1}$ COPPE, Universidade Federal do Rio de Janeiro, RJ, Brasil \\ ${ }^{2}$ Instituto de Física, Universidade Federal do Rio de Janeiro, RJ, Brasil \\ ${ }^{3}$ Laboratório de Biomembranas e Redes Neurais, \\ Universidade Estadual do Rio de Janeiro, RJ, Brasil \\ Recebido em 15/04/04; Aceito em 26/05/04
}

\begin{abstract}
Neste trabalho propomos um sistema de medida e coleta de dados para trilhos de ar baseado em sensores magnéticos ligados à porta de jogos de um PC. A leitura dos dados na porta é feita por um pequeno programa escrito em Logo. O sistema foi testado com excelentes resultados em um estudo dos movimentos uniforme e uniformemente variado sobre o trilho.
\end{abstract}

Palavras-chave: $\log$, trilho de ar, uniforme, uniformemente variado.

In this work we propose a measure system and collects data for air's trails using magnetic sensor linked to the game port of a PC. The reading of the data in the door is made by a small program written LOGO language. The system was tested with excellent results in studies of the uniform movement and evenly varied.

Keywords: air's trail, uniform movement, evenly varied.

\section{Introdução}

Um problema comum na montagem de experimentos de mecânica é a eliminação de forças de atrito indesejadas. Nos laboratórios didáticos isto é freqüentemente realizado com o auxílio de trilhos de ar (a construção de alguns sistemas desse tipo está descrita nas Refs. [1, 2]). Mesas de ar [3] ou discos com gelo seco [4] são empregados quando movimentos em duas dimensões são estudados. O uso do trilho de ar quase sempre requer um aparato que meça a posição em função do tempo do carrinho que desliza sobre ele. Geralmente isto é feito com centelhadores que marcam uma fita de papel, ou com "photogates" ligados a um cronômetro digital ou computador.

Neste trabalho apresentamos um sistema de medida e coleta de dados para trilhos de ar que pode substituir com vantagens os centelhadores e photogates. Ele é baseado em um sensor de proximidade muito simples e de custo extremamente baixo, o "reed-switch" [5], acoplado à porta de jogos (a entrada do "joystick") de um PC. A porta é lida com um pequeno programa escrito em Logo, nos moldes descritos na Ref. [6]. Na próxima seção descreveremos em mais detalhe o sistema de medida e aquisição de dados, e sua montagem sobre um trilho de ar eficiente e de fácil construção. $\mathrm{Na}$ Seção 3 mostraremos que o sistema se comporta muito bem em experimentos típicos de um laboratório escolar. Na Seção 4 apresentaremos nossas conclusões.

\section{Descrição do sistema}

O modelo de trilho de ar utilizado neste trabalho é semelhante ao apresentado na Ref. [1], e é feito com tubos e conexões de PVC usados em construção civil. O trilho é apoiado sobre uma base de madeira, um pouco acima e paralelo a um perfil de alumínio em U onde são fixos os sensores de posição. Os detetores são ligados a dois bornes que serão conectados à porta de jogos. $\mathrm{O}$ ar injetado no trilho sai de um aspirador de pó doméstico. A montagem está mostrada na Fig. 1.

O movimento do carrinho sobre o trilho é monitorado por um conjunto de chaves magnéticas chamadas de reed-switch ou reed-relay. A Fig. 2 mostra o aspecto de um desses sensores. Em geral ele

\footnotetext{
${ }^{1}$ Enviar correspondência para Francisco Laudares. E-mail: f_laudares@ hotmail.com.
} 
é apresentado como uma pequena ampola de vidro que contém em seu interior duas lâminas de material ferromagnético bem próximas uma da outra. Se aproximamos um imã do reed-switch as duas lâminas se encostam, fechando os contatos externos. O sensor funciona como uma chave ativada por campos magnéticos. $\mathrm{O}$ estado desta chave (aberta/fechada) pode ser facilmente lido pela porta de jogos de um PC, bastando para isto que se ligue o reed-switch aos pinos correspondentes a um botão do joystick, que também opera como uma chave de contato [6]. Com isto, medidas de tempo feitas com o relógio de computador podem ser associadas a passagens pela posição do reed-switch.

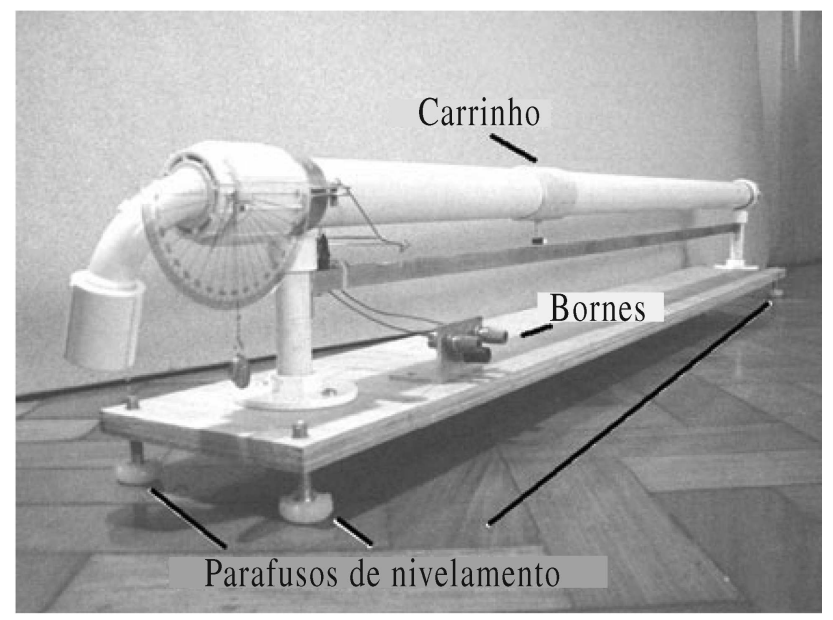

Figura 1 - Trilho de ar usado para o experimento. O transferidor e o fio de prumo medem a inclinação do trilho.

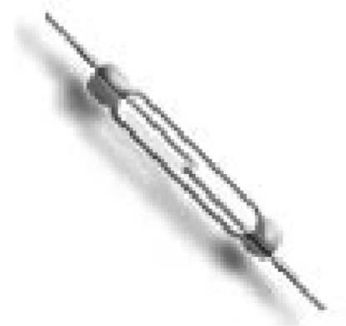

Figura 2 - Um reed-switch.

O carrinho utilizado no trilho é mostrado na Fig. 3, é construído com um pedaço de tubo de PVC de diâmetro ligeiramente maior que o do trilho, com um acabamento na face interna para facilitar o deslizamento. Um imã é fixado ao carrinho, com o auxílio de uma pequena haste, e serve para acionar os sensores magnéticos durante o movimento do carrinho pelo trilho.

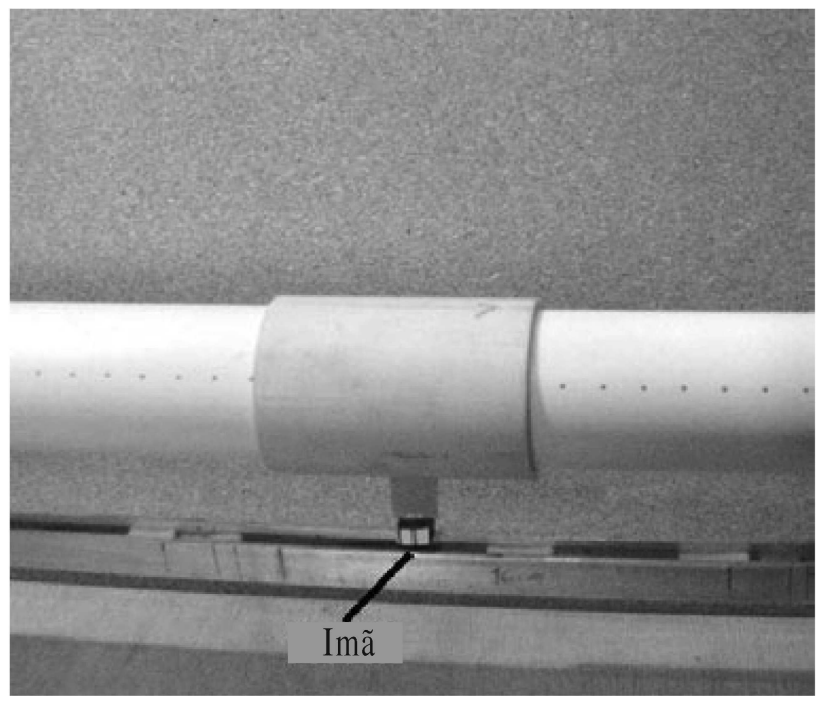

Figura 3 - Carrinho com o imã acoplado.

Colocamos sete sensores no perfil de alumínio sob o trilho, separados de $16 \mathrm{~cm}$ um do outro, completando $96 \mathrm{~cm}$ entre o primeiro e o último. Os detetores são ligados em paralelo aos dois bornes do trilho, que por sua vez são conectados à porta de jogos na posição correspondente a um botão do joystick [6]. Assim, quando o imã passar por um reed-switch, suas lâminas fecham contato e a porta de jogos recebe um sinal de "botão apertado". A porta é lida por um pequeno programa escrito em Logo, que registra os instantes em que os sensores magnéticos são acionados pela passagem do carrinho com o ímã. Mais detalhes sobre o funcionamento da porta de jogos e como ela pode ser lida por programas Logo estão na Ref. [6].

\section{Experiências com o sistema}

Para testar o sistema de medida e aquisição de dados, realizamos dois experimentos com o trilho de ar: estudamos o movimento do carrinho quando o trilho estava perfeitamente horizontal, e quando ele tinha uma certa inclinação. No primeiro caso, em que a força resultante sobre o carrinho é nula, deixamos o trilho de ar na horizontal ajustando os parafusos de nivelamento da base (ver Fig. 11) até que o carro só se mova com um empurrão. O movimento do carrinho após um empurrão é mostrado na Fig. 4 Uma reta

$$
x=x_{0}+v_{0} t+\frac{1}{2} a t^{2}
$$

foi ajustada por mínimos quadrados aos pontos experimentais, resultando em $x_{0}=-0,0003 \pm 0,0001 \mathrm{~m}$ 
e $v_{0}=0,6859 \pm 0,0002 \mathrm{~m} / \mathrm{s}$. A qualidade do ajuste pode ser avaliada na Fig. 4 e mostra que o movimento é uniforme, ou seja, a velocidade do carrinho sobre um trilho horizontal é constante.

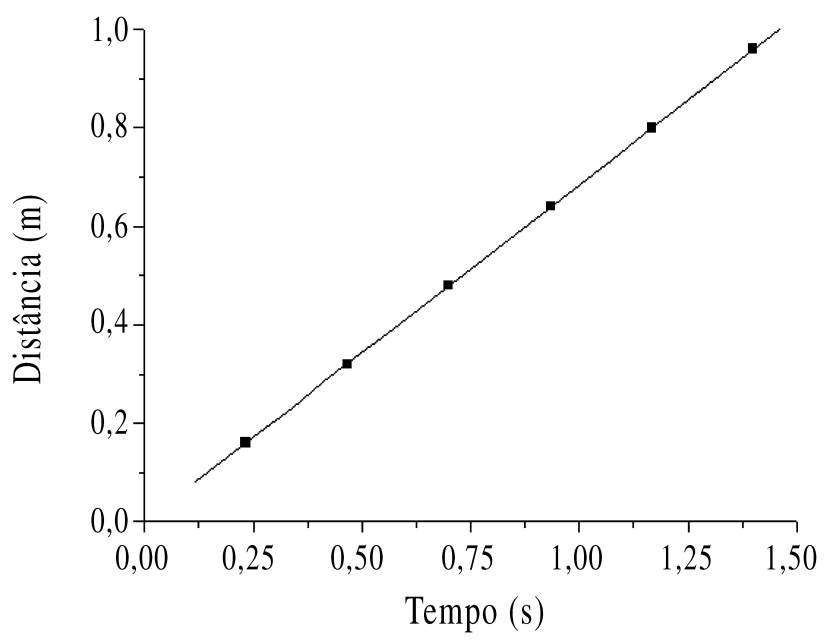

Figura 4 - Distância em função do tempo no trilho horizontal.

No Ensino Médio, ajustes de curva não são ferramentas comuns. A uniformidade do movimento pode, então, ser observada a partir do cálculo direto da velocidade. Na Fig. 5 mostramos as velocidades médias entre detetores sucessivos, calculada com os pontos da Fig. 4 Vemos que estas velocidades são praticamente idênticas.

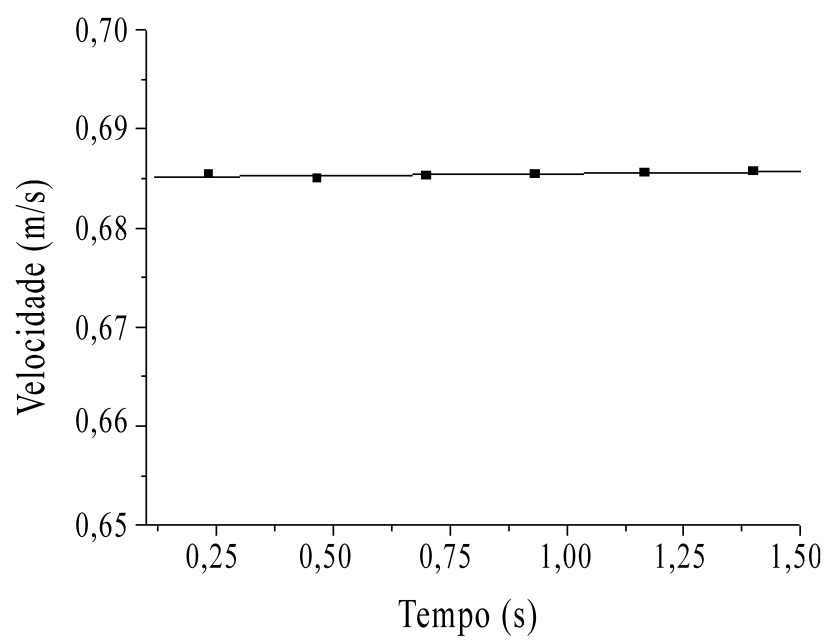

Figura 5 - Velocidades médias no trilho horizontal.

No segundo experimento, o trilho foi inclinado com um ângulo de aproximadamente $2^{\circ}$ para que a resultante da força no carrinho não seja nula. O movimento do carrinho abandonado no trilho está mostrado na Fig. 6

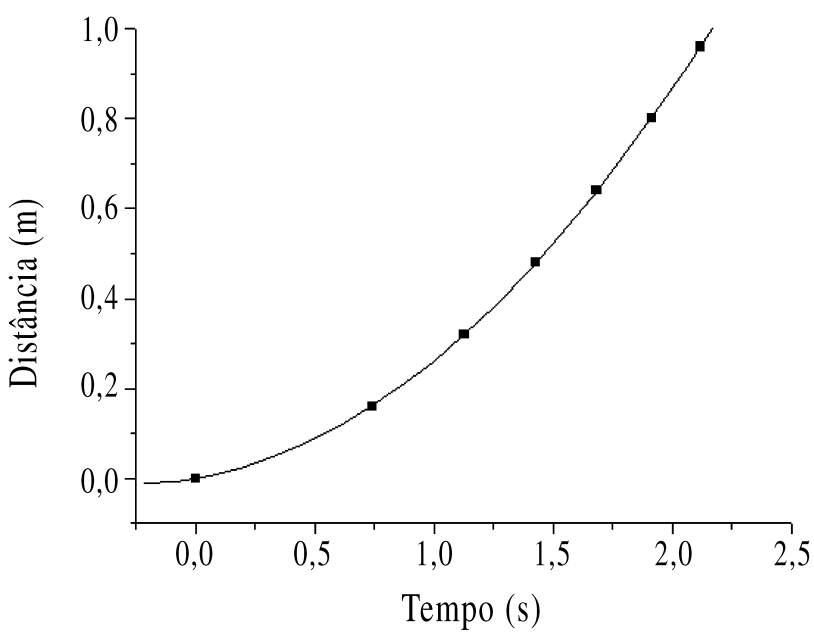

Figura 6 - Distância em função do tempo no trilho inclinado.

Ajustando a parábola

$$
x=x_{0}+v_{0} t+\frac{1}{2} a t^{2}
$$

aos pontos medidos, obtemos $x_{0}=-0,013 \pm 0,009 \mathrm{~m}$, $v_{0}=0,09 \pm 0,01 \mathrm{~m} / \mathrm{s}$ e $a=0,34 \pm 0,01 \mathrm{~m} / \mathrm{s}^{2}$. A Fig. 6 mostra a qualidade do ajuste, comprovando que no trilho inclinado o movimento é uniformemente acelerado.

Desprezando os atritos, a aceleração $a$ do carrinho no trilho é dada pela relação bem conhecida

$$
a=g \operatorname{sen} \alpha
$$

onde $g$ é a aceleração gravitacional e $\alpha$ o ângulo entre o trilho de ar e a horizontal. Substituindo nesta equação os valores de $a$ e $\alpha$ dados acima, obtemos para a aceleração da gravidade

$$
g=9,7 \pm 0,3 \mathrm{~m} / \mathrm{s}^{2}
$$

A velocidade média entre os detetores está mostrada na Fig. 7 onde podemos observar que ela 
aumenta linearmente com o tempo.

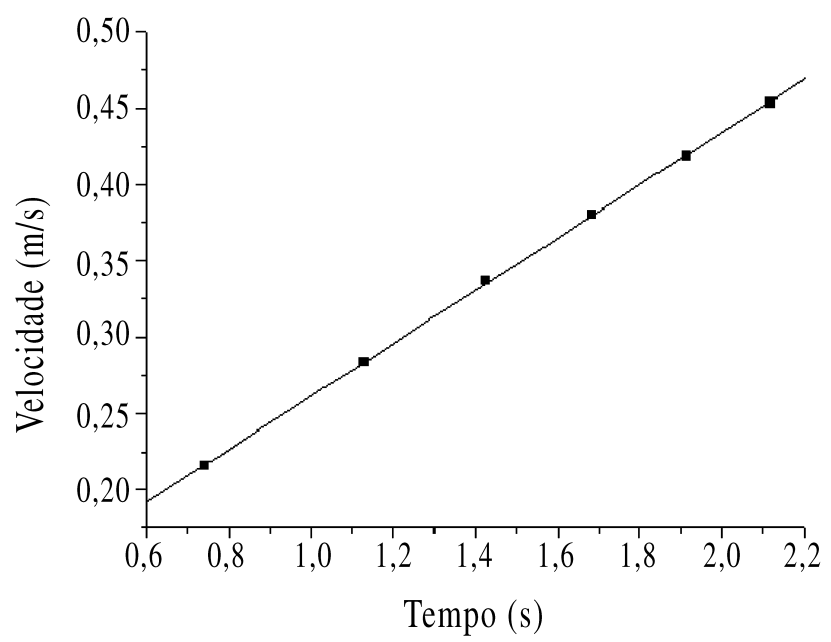

Figura 7 - Velocidades médias no trilho inclinado.

Investigamos também como a aceleração do carrinho depende da inclinação do trilho. Medidas da aceleração em função de sen $\alpha$, para quatro ângulos $\alpha$ diferentes, estão mostradas na Fig. 8

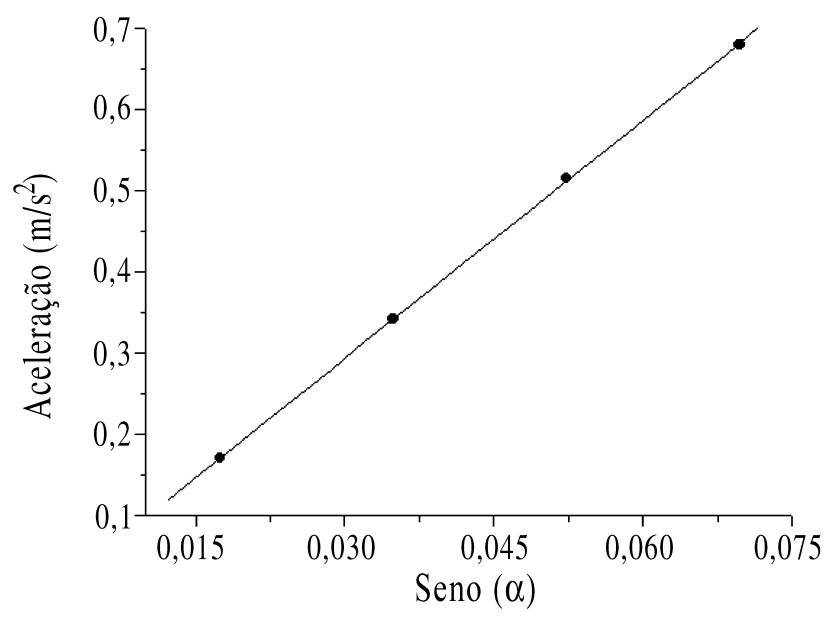

Figura 8 - Aceleração em função do seno do ângulo de inclinação.

Vemos na Fig. 8 que a aceleração depende linearmente de sen $\alpha$, como esperado da equação (3). Ajustando uma reta aos pontos medidos obtemos para a aceleração gravitacional (o coeficiente angular da reta)

$$
g=9,77 \pm 0,07 \mathrm{~m} / \mathrm{s}^{2}
$$

um resultado é bastante razoável, e bem mais preciso que a estimativa anterior (Eq. (4). O valor de $g$ no Rio de Janeiro é $9,788 \mathrm{~m} / \mathrm{s}^{2}$.

\section{Comentários finais}

Mostramos neste trabalho que o reed-switch, um sensor pouco encontrado nos laboratórios didáticos, pode ser utilizado para medir posições sobre um trilho de ar (veja também a Ref. [1]). Quando acoplado à porta de jogos de um PC, como se fosse um botão de joystick, o reed-switch permite que se façam medidas de posição e tempo de qualidade comparável à dos métodos usuais, com um custo muito baixo e uma grande facilidade de montagem e operação. Nós testamos o sistema comprovando que na ausência de forças (trilho horizontal) o movimento é uniforme, e que na presença de uma força constante (trilho inclinado) ele é uniformemente acelerado. Realizamos ainda uma verificação experimental da relação entre aceleração e ângulo em um plano inclinado, extraindo daí uma medida bastante precisa da aceleração da gravidade. Essas experiências, típicas de um curso introdutório de física experimental, mostram que o sistema proposto pode ser usado com sucesso em laboratórios didáticos.

\section{Agradecimentos}

Agradecemos ao Prof. Carlos Eduardo Aguiar, do Instituto de Física da UFRJ, pelas muitas discussões e sugestões.

\section{Referências}

[1] J.R. Pimentel, V.H. Zumpano e L.T. Yaginuma, Revista de Ensino de Física 11, 15 (1989).

[2] H.V. Neher and R.B. Leighton, American Journal of Physics 31, 255 (1963).

[3] J.R. Pimentel, Caderno Catarinense de Ensino de Física 12, 150 (1995).

[4] R. Hessel, Revista de Ensino de Física 4, 3 (1982).

[5] M.M. Werneck, Transdutores e Interfaces (LTC, 1996), p. 92.

[6] C.E. Aguiar e F. Laudares, Revista Brasileira de Ensino de Física 23, 371 (2001). 